\title{
Variations
}

Variations

Revue internationale de théorie critique

$17 \mid 2012$

Critique du travail

\section{Trente-trois thèses et une question}

\section{Dietrich Hoss}

\section{OpenEdition}

Journals

Édition électronique

URL : http://journals.openedition.org/variations/387

DOI : 10.4000/variations.387

ISSN : 1968-3960

Éditeur

Les amis de Variations

Référence électronique

Dietrich Hoss, «Trente-trois thèses et une question », Variations [En ligne], 17 | 2012, mis en ligne le 15 octobre 2012, consulté le 22 septembre 2020. URL : http://journals.openedition.org/variations/387 ; DOI : https://doi.org/10.4000/variations.387

Ce document a été généré automatiquement le 22 septembre 2020.

Les ami•e•s de Variations 


\title{
Trente-trois thèses et une question
}

\author{
Dietrich Hoss
}

\section{RÉFÉRENCE}

John Holloway, Crack capitalism, 33 thèses contre le capital, Paris, Editions Libertalia, 2012, $458 \mathrm{p}$.

1 Il vient de paraître en français un deuxième livre de John Holloway : Crack capitalism. 33 thèses contre le capital (Editions Libertalia 2012, 464 p. 20 Euros). Son précédent livre Changer le monde sans prendre le pouvoir, le sens de la révolution aujourd'hui dont la traduction française apparut en 2007 avait trouvé en France moins d'écho que sur la scène internationale en général. Comme l'expliquent l'éditeur et le traducteur dans leur introduction du nouveau livre, ce n'est pas seulement Holloway mais c'est toute une constellation intellectuelle dans laquelle s'inscrit l'auteur, associée à des approches nommées « marxisme ouvert, critique de la valeur, théorie critique », qui est largement ignorée ici, un espace de réflexion et d'échange où se situe par contre «Variations » qui a publié des textes de Holloway à plusieurs reprises. Les courants de l'univers intellectuel prédominant que Holloway appelle « le marxisme traditionnel » qui s'étend entre une gauche établie, parlementariste, et la gauche de cette gauche attachée à ses variantes plus radicales, sont tous très loin d'une perspective qui vise à "changer le monde sans prendre le pouvoir ". S'ils ne sont pas dans une perspective de conquête de positions gouvernementales par des voies parlementaires, à la rigueur appuyés par des mobilisations extra-parlementaires, ils cherchent au moins à conquérir une position de contre-pouvoir dans l'espoir d'exercer une pression sur l'Etat pour que celui-ci arrive à imposer des freins à la domination capitaliste et sa destructivité. Espérons que le nouveau livre de Holloway rencontre dans le contexte actuel un nouveau public plus ouvert à une mise en question de ces vieux schémas. La crise dite financière a provoqué des mouvements massifs, spontanés, avec de nouvelles interrogations et formes de lutte contre la tentative de créer les conditions pour un nouveau cycle d'accumulation basé sur la détérioration extrême des conditions de vie des populations. Mais ces mouvements aussi risquent de perdre leurs énergies soit dans des mobilisations pour 
des revendications plus ou moins conventionnelles dirigées aux pouvoirs nationaux ou européens, soit dans des actions pseudo-insurrectionnalistes de combats de rue.

2 Le nouveau livre de Holloway ne constitue pas seulement une ouverture de perspectives face à ces fausses alternatives, mais aussi face à des positions critiques plus adéquates qui analysent la crise actuelle du capitalisme comme conséquence de son fonctionnement sur la base des rapports sociaux fétichisés - comme valeur, argent, capital, marchandises - et l'impossibilité de sa domestication par l'action étatique, mais qui le font d'une façon unidimensionnelle c'est-à-dire sans montrer les failles, les contradictions, qui pourraient constituer une chance pour le dépassement de cette régulation sociétale de plus en plus mortifère pour l'homme et son environnement. L'identification du potentiel de ces failles et contradictions, appelées par Holloway aussi brèches et fissures dans le capitalisme, qui pourront in fine faire craquer le capitalisme, le remplacer par une nouvelle synthèse sociale, est la tâche de ce nouveau livre. Mobilisant un riche fonds de travaux théoriques, principalement constitué par une relecture de Marx à la suite des auteurs de l'Ecole de Francfort, et analysant d'une façon détaillée les expériences de luttes de dernières années, il cherche à montrer l'antagonisme fondamental entre la dynamique d'expansion et d'accumulation du capital et les résistances, mécanismes d'auto-défense et combats des hommes et des femmes au quotidien. Comme dans son premier livre, sa pensée se caractérise par une démarche qui arrive à fluidifier les catégories abstraites, pas seulement les catégories d'une science sociale et économique plus ou moins positiviste, mais aussi les concepts relativement abstraits déjà chez Marx, mais rigidifiés et rendus finalement méconnaissables par leur usage dogmatique "marxiste-léniniste». Il montre qu'endessous de l'apparence d'une constellation figée, pétrifiée, imperturbable, la domination du monde par les fétiches (capital, argent, marchandise, etc.), il est forcément déployé un effort, renouvelé en permanence, pour reproduire et recréer cet ordre fétichiste. Il en donne un exemple :

3 « Des menaces de violences requises actuellement pour produire et reproduire la séparation entre les producteurs et les moyens de production sont peut-être beaucoup plus importantes aujourd'hui que tout ce que Marx pouvait même imaginer. L'enclosure de la terre et le respect de la propriété privée requièrent une immense armée pour s'imposer. Si nous ne comptons pas seulement les gardiens de sécurité, les policiers, les militaires mais aussi les juristes, les travailleurs sociaux et les enseignants (sans parler des parents), alors une partie très significative de la population mondiale est engagée dans la séparation constamment réitérée des gens de leurs moyens de production.» (p.273)

4 Le règne du fétichisme est impensable sans un processus de fétichisation permanent. Le plus important est donc d'avoir une idée claire du substrat de ce processus, de se rendre compte de ce qui est fétichisé : des rapports sociaux occultés et objectivés dans l'argent et la marchandise. Ceux-ci sont rendus visibles et revitalisés par Holloway à travers leur fluidification conceptuelle sous forme d'une transformation grammaticale : le substantif «rapports sociaux » adopte une forme verbale, il devient «le faire » des hommes produisant la réalité. De cette manière, il aide à comprendre le constat de Marx qui avait découvert que ce «faire », que lui-même appelait dans les Manuscrits de 1844 l'« activité vitale consciente » des hommes, subit dans le capitalisme une mise en forme qui lui donne une « double nature ». D'un côté cette activité est transformée en travail abstrait, absorbée comme une activité sans qualité, mesurée comme temps de 
travail socialement nécessaire pour produire une marchandise, pure dépense de l'énergie humaine pour créer de la valeur, de la plus-value, sédimentée en argent et capital. De l'autre côté, l'activité de l'homme reste une activité concrète, utile, produisant la richesse des valeurs d'usage. Tout le livre de Holloway est centré autour de ce double caractère dans le contexte sociétal actuel de l'activité humaine qu'il préfère appeler « le faire », car plus large que l'activité productrice dans les cadres du travail, se développant « dans-contre-et-au-delà » du travail, comme il dit. Marx luimême et les théoriciens qui cherchaient à prolonger et à actualiser son approche se sont concentrés presque exclusivement sur l'analyse détaillée des processus économiques et sociaux basés sur cette transformation de l'activité en travail entraînant pour l'humanité une fabuleuse augmentation de ses capacités scientifiques et techniques de transformation du monde mais également une scandaleuse disparité des conditions de vie, de crises et de destructions massives.

Holloway met l'accent sur l'autre côté de l'activité humaine, sa dimension non commensurable par la loi de la valeur, résistante à l'abstraction et à l'absorption par le capital. Cette dimension non contrôlée du « faire » des millions d'hommes et de femmes crée autant de brèches, fissures et fuites dans le fonctionnement du capitalisme. Les exemples qu'il en donne dans un inventaire succinct sur trois pages se situent entre « la jeune fille de Tokyo qui dit qu'elle n'ira pas au travail aujourd'hui et va s'asseoir dans un parc pour lire son livre... ", « l'ouvrier de l'automobile à Birmingham qui se rend le soir à son jardin ouvrier afin d'avoir une activité ayant un sens et lui procurant du plaisir » et "ce jeune homme à Mexico qui, exaspéré par la brutalité du capitalisme, part à la jungle pour organiser une lutte armée pour changer le monde.» (p. 24 ss.) $C^{\prime}$ 'est ce potentiel de résistance, cette manifestation d'une aspiration à une vie digne et autodéterminée, qui est pour Holloway à la base de la crise actuelle du capitalisme, qui l'empêche d'aller en avant dans sa course effrénée d'expansion destructive. En même temps, en partant de l'antagonisme constitutif entre travail abstrait et le "faire " Holloway évite de construire de nouvelles abstractions et mystifications à la Hardt et Negri qui structurent la réalité sociale comme un combat entre des nouvelles entités réifiées: «empire" contre "multitude». Le capitalisme reste pour Holloway un mécanisme de structuration aveugle, un "sujet automate " comme disait Marx, non maîtrisé ni par une catégorie sociale -le capital financier, les multinationales..., ni par des institutions politico-économiques -la Banque mondiale, le FMI, les G7, 8,20... Les brèches de leur côté, les tentatives d'une variété infinie des hommes et des femmes à créer des espaces où ils peuvent respirer, prendre au moins partiellement leur vie en main, sont toujours incertaines et précaires. Elles sont toujours en danger d'être annulées, perverties, par «la succion gélatineuse de la synthèse capitaliste » (p.94), c'est-à-dire de devenir simplement « des moyens pour résoudre les tensions et les contradictions du capitalisme.» (p.98) Elles sont confrontées à l'action de l'Etat qui cherche à les liquider là où il est incapable de les instrumentaliser pour renouveler son contrôle sur la société. Elles affrontent la loi de la valeur qui impose un comportement conforme aux règles marchandes pour survivre. Et surtout elles sont confrontées à des obstacles intérieurs. Les «contradictions du capitalisme se reproduisent au sein de notre révolte ": "même les groupes qui mettent en avant dans leur programme la création de relations sociales différentes entre leur membres finissent parfois en querelles amères et avec un fort sentiment de désillusion. Parfois l'intensité de l'effort pour créer quelque chose de différent se reflète dans l'amertume intense d'animosités qui se sont créées. » (p. 119) Il est extrêmement difficile de se libérer du carcan dans 
lequel nous sommes renfermés, chacun à sa manière, dans le capitalisme. «Le travail abstrait enferme à la fois nos corps et nos esprits.» (p.185) L'abstraction du travail «brise l'être-social-qui-fait, le nous, en une multiplicité de sujets individuels... » (p.189) Alors le seul espoir réside pour Holloway dans la possibilité « d'élargir les brèches, de les multiplier et de favoriser leur convergence » (p.35) pour déclencher une dynamique dirigée contre cet isolement atomisant.

6 Le déclenchement d'une telle dynamique nécessite selon lui la création d'un nouveau langage de la lutte, de nouveaux principes d'organisation qui rompent d'avec les schémas traditionnels d'un regroupement du prolétariat, de la classe ouvrière, ou plus actuel - des femmes, des "minorités », des «sans ... ». Toutes ces catégorisations sociales visant illusoirement à identifier un sujet historique de transformation ne servent qu'à créer des entités qui restent dans l'enceinte de la reproduction du modèle régnant de structuration des rapports sociaux. Elles renferment les hommes et les femmes en lutte dans des identités constitutives du mécanisme économique et politique, elles renvoient à des situations de classe, elles «class-ifient » les luttes: les travailleurs luttent pour un emploi stable et une meilleur rémunération en tant que travailleurs, les femmes pour leurs droits en tant que femmes, les «sans... " pour leur intégration en tant que groupes sociaux défavorisés ou exclus. Alors face à ce formatage des luttes faut-il «trouver des manières de rendre visibles et de renforcer [des] lignes de continuité » (p.72), développer de "formes d'organisation noninstrumentales ... [qui] agissent vers un au-delà plutôt qu'en retrait par rapport au but qui doit être atteint... » (p.82) Car finalement « au-delà des buts immédiats et bien plus que leur succès (ou pas), ce qui reste crucial [dans les luttes] est la création ou la redécouverte de relations sociales différentes.» (p.84) Ce sont des relations comme « camaraderie, dignité, amorosité, amour, solidarité, fraternité, amitié, éthique » qui se « dressent en opposition avec les rapports marchands et monétaires du capitalisme »... « des rapports se développant dans les luttes contre le capitalisme et qui peuvent être vu comme anticipant ou créant une société au-delà du capitalisme.» (p.86) Bien sûr ce genre de relations non-marchandes émergeaient aussi dans le cadre des organisations traditionnelles du mouvement ouvrier. Mais elles étaient considérées comme un « sousproduit de la lutte. » Elles devaient juste servir à atteindre les objectifs immédiats voire la conquête du pouvoir dans le futur. Dans la nouvelle orientation d'une politique préfigurative postulée par Holloway, elles deviennent l'objectif même de la lutte : « la lutte pour une société différente doit créer cette société à travers ses formes de luttes. »(p.89)

7 Du reste ce livre relativement volumineux constitue une analyse détaillée de la dynamique à l'œuvre de l'antagonisme entre le travail abstrait et le «faire » à notre époque, en temps de crise. Ce sujet qui pourrait paraître théorique et abstrait est toujours traité d'une façon très concrète en référence à des exemples de luttes variés, dans un langage direct, à la première personne, plein de métaphores, qui rend la lecture accessible et stimulante. L'interrogation à propos des thèses avancées par Holloway qui va suivre ne fait que prolonger le fil de ses réflexions comme lui-même l'avait voulu, définissant sa démarche comme « une tentative de créer des concepts ouverts qui questionnent plutôt que d'instaurer un paradigme...» (p.38), selon la devise zapatiste : «En nous interrogeons nous marchons (preguntando caminamos)».

8 Alors récapitulons: "L'activité vitale consciente», "le faire», des hommes et des femmes mise en œuvre pour enrichir leur vie en commun est captée, atrophiée, 
frustrée, par son conditionnement et sa transformation en travail abstrait, en valeur, source de plus-value et d'un capital qui cherche à imposer coûte que coûte à la société dans le monde entier ses nécessités de reproduction et d'accumulation ravageuses. Mais celui-ci de son côté se heurte à la volonté de survie et à l'aspiration d'une vie digne autodéterminée de millions d'êtres humains qui créent des brèches dans la structuration sociétale par le capital. Ces brèches restent pourtant précaires, exposées à des récupérations et des instrumentalisations de restabilisation du système. Elles restent exposées à des obstacles extérieurs et intérieurs, principalement parce qu'elles sont isolées les unes des autres, elles s'ignorent - ou pire - se méprisent. Face à cette situation Holloway lance l'appel à la continuation de créer ces brèches : «...faisons-les se déployer, se multiplier, résonner entre elles, faisons-les converger.»(p.425) Mais c'est là où se pose le problème : si on peut supposer que les brèches vont continuer de se créer plus ou moins spontanément, avec une certaine résonance entre elles - des fois même d'une façon explosive, massive, comme en Grèce, au Maghreb, en Espagne et ailleurs dans les dernières années - ce que demande un effort conscient plutôt organisé c'est la création de liens qui les stabilisent, approfondissent et les font converger. Il me semble donc que c'est sur l'initiation et la pérennisation d'une réflexion, d'une pensée en commun, qu'il faut se concentrer en priorité dans la constellation actuelle. Holloway parle d'un tel décloisonnement du penser s'il dit : « Le savoir, qui est un élément de ce faire, fait partie de ce même mouvement [du faire contre-et-au-delà du travail] : c'est aussi un dégel, un flux social qui brise les définitions, un flux dans lequel le savoir d'une personne se mêle et se mélange de façon indéfinissable au savoir des autres. » (p.414) Mais il voit ce dégel plutôt comme un processus en cours - «Il y a (...) un partage continuel d'idées et d'informations, (...) le tissage continuel d'un nous, le façonnage d'un flux commun de faire et de rébellions » (p.416) - et moins comme une tâche à accomplir. L'intensification et l'accélération de ce processus n'est pas sa préoccupation centrale.

9 En réalité c'est contre ce dégel du savoir - qu'il faudrait mieux appeler le dégel du penser - qu'est dirigé non seulement l'encadrement de la formation scolaire et universitaire qu'il évoque en disant: «Brisons les murs autour de notre esprit qui rigidifient notre pensée, un processus qui résulte du travail abstrait et qui est renforcé dans les écoles et les universités. » (p.424) C'est tout ce gigantesque appareillage de l'industrie culturelle, constitutif de la "société du spectacle », perfectionné à un degrés inouï les dernières décennies avec l'arrivée des instruments multimédiaux, fermant avec son avalanches des images et de sons tous les pores d'un temps potentiellement libre pour le penser et l'imagination. Holloway ne mentionne nulle part ce monstre qui est pourtant l'agent principal pour faire accepter le règne du travail abstrait, de la valeur, de l'argent et de la marchandise. L'aspiration à un autre faire, à la jouissance et au rêve, est utilisée, canalisée et défigurée comme carburant à cette machinerie. Et même si Holloway cite maintes fois Adorno et Marcuse, il ne fait pas référence à leur analyse de l'ancrage de ce mécanisme dans la structure pulsionnelle ni aux effets d'une pseudo-libération $\mathrm{du}$ principe de plaisir, appelée par Marcuse "désublimation répressive ". C'est seulement par ce point aveugle que s'explique le fait que Holloway considère par exemple les raves parties comme une brèche dans le capitalisme (p.117) à la place de les comprendre comme un moyen de l'industrie culturelle pour transformer les désirs de jeunes de se regrouper, de sortir de leur isolement, de créer un espace autodéterminé, en un étourdissement par la violence du bruit conduisant à 
l'annihilation du penser et de l'échange, à la constitution d'une variante spécifique d'une « foule solitaire».

Peut-être est-ce la domiciliation de Holloway en Amérique Latine, où il enseigne depuis une vingtaine d'années, qui explique une certaine sous-estimation de l'impact de l'industrie culturelle dans les métropoles du capitalisme mondial. Les exemples de luttes collectives et débats politiques auxquels il se réfère sont principalement localisés dans cette région, dans un contexte socio-culturel où le contrôle social s'exerce d'une façon plus immédiate, par l'appareil répressif étatique et les pouvoirs caciques et mafieux. Une fois ces forces neutralisées peuvent se libérer les énergies et l'inventivité pour créer de nouvelles formes de rapports sociaux: zapatistas, piqueteros, mouvements d'occupations de terres... Sur cet arrière fond peut se développer un débat relativement large, ouvert, sur les implications théoriques de ces mouvements - même dans le cadre institutionnel universitaire. Chez nous, dans les métropoles, les formes réifiées de la pensée et « le voile idéologique » (Adorno) couvrant la réalité sont ancrés d'une façon beaucoup plus perfectionnée dans les consciences, aussi bien dans les milieux du penser professionnel, académique et médiatique, que dans la population en général à travers l'industrie culturelle. Tout est fait pour empêcher le penser en général et pour étouffer, marginaliser, déformer ou récupérer une pensée radicale déviante en particulier. Ceci d'autant plus que la contradiction entre un niveau du " general intellect ", scientifique et culturel, jamais atteint et l'enserrement de celui-ci dans les structures du travail abstrait est toujours plus aigüe. La conséquence est l'épuisement, la résignation ou le repli sectariste de la pensée critique. Dans ce contexte l'appel que dirige vers nous Holloway de créer et faire converger de brèches dans le capitalisme, tout juste qu'il soit, a quelque chose de bien intentionné mais inoffensif. Dans une situation où toute issue pour éviter l'accomplissement du désastre final semble bétonnée, où toute perspective d'un changement véritable est rendu impensable - « il est plus facile de penser la fin du monde que la fin du capitalisme » comme dit Holloway lui-même - est-ce qu'il ne faut pas reformuler son propos en nous demandant comment pourrions-nous débloquer ce verrouillage du penser si parfait? Comment rendre l'impensable de nouveau pensable? Est-ce qu'il ne faut pas concentrer tout l'effort sur cette tâche, la libération de cette dimension du " faire » qui est le penser? Ce combat semble dans la situation actuelle le plus difficile mais en même temps le plus nécessaire. Vu la pression énorme qui s'exerce à l'extérieur et à l'intérieur de l'individu contre un tel éveil d'un penser imaginatif il faut envisager des armes plus lourdes qu'un simple appel à la désertion générale - si juste et convaincant soit-il - lancé par Holloway. Puisque les capacités de résistance de l'individu seul sont limitées s'impose la recherche de nouvelles formes d'union et d'association pour nous rendre capable de déployer ensemble nos forces réflexives et imaginatives. Il est vrai, les formes traditionnelles du cadre organisationnel d'une pensée révoltée collective l'organisation révolutionnaire, le Parti - sont caduques. Elles étaient d'ailleurs toujours controversées car sujettes au risque de dérives monstrueuses. Holloway se dirige donc avec raison contre toute réanimation du modèle d'organisation léniniste. Mais est-ce qu'on doit à cause de cela abandonner l'idée de la construction d'un espace commun, autonome, aux niveaux local, national, international, de débat et d'entendement sur les perspectives d'une sortie du capitalisme, un espace où le "nous ne savons pas » serait " un principe d'organisation qui vise à la participation de tout le monde au processus de détermination de notre faire individuel et collectif » comme définit Holloway, l'alternative aux structures organisationnelles traditionnelles qui étaient basées sur le 
principe du savoir des dirigeants. (p.415) Est-ce que la construction d'un tel espace se fera tout seul, sous des formes spontanées, ou - vu que tout s'y oppose - ne faut-il pas développer un effort systématique pour créer de passerelles, des synergies, des transparences entre les différentes « brèches " pratiques et théoriques, pour y arriver? Holloway indique en passant, quasiment hors champ de son texte, dans les « remerciements » à la fin de son livre les contours de l'espace d'un penser en commun qui a permis le développement de sa pratique théorique à lui. Il dit que son livre, " comme tout faire, fait partie du flux social et non grammatical, où le faire, le vécu, l'amour de l'un se jette dans le faire, le vécu et l'amour des autres... » (p.427) Les dimensions de cet espace de confluence des apports et résonances qu'il évoque sont multiples, le " vortex » de sa compagne, les débats avec ses amis, collègues et étudiants dans et dehors des institutions universitaires à l'université de Puebla et en Angleterre, de groupes de travail à Buenos Aires et surtout de "milliers de personnes qui ont participé aux discussions sur la mère de ce livre, Changer le monde sons prendre le pouvoir, qui m'ont immergé dans un monde de débats théoriques et pratiques.»(p.429) Holloway s'est construit un tel espace de convergence et d'élaboration d'un penser nouveau à sa mesure en effet quasi spontanément, utilisant à fond les possibilités d'un enseignant-chercheur engagé de renommé international. Mais «la jeune fille dans le parc de Tokyo ", "l'ouvrier de l'automobile à Birmingham qui se rend le soir à son jardin ouvrier" , tous les millions d'hommes et de femmes qui créent selon Holloway des brèches dans le capitalisme sans le savoir, et même tous les groupes et individus qui le font ou veulent le faire consciemment mais qui restent isolés les uns des autres, eux n'ont pas la possibilité de créer spontanément un tel espace de rencontres, d'échange, de libération et de développement d'un penser en commun.

En fait, elles commencent à émerger déjà de telles structures aujourd'hui de mille façons: blogs et sites de groupes et d'individus invitant au débat, "causeries " publiques avec des auteurs autour de leurs livres, débats ouverts variés dans des clubs, des cafés et des librairies, séminaires, groupes de travail, « universités autonomes » et universités d'été... Mais ces initiatives sont exposées, comme toutes les brèches citées par Holloway, à des obstacles extérieurs et intérieurs : les blocages et l'étouffement ambiant du penser sont reproduits dans les milieux militants comme hostilité à la théorie, déclarée per se élitiste et dominatrice, détournant de l'action. Le comportement individualiste et concurrentiel intériorisé comme règle de jeu pour la survie dans le contexte économique et social régnant est reproduit comme une attitude sectaire que Holloway illustre bien en disant : «Créer des rigidités, des dogmes et des "nous ne leur parlons pas parce qu'ils sont réformistes", "nous n'avons rien à faire avec eux parce qu'ils boivent du Coca-Cola", "nous ne coopérons pas avec eux parce qu'ils sont sectaires", c'est prendre une part active dans le gel du flux des rébellions, c'est reproduire les définitions, les classifications et les fétiches de la pensée capitaliste. » (p. 418) Le résultat est un éclatement, une ignorance des uns des autres et une invisibilité générale de tous ses efforts parfois intellectuellement considérables et pertinents. Rien ne semble plus difficile à développer qu'un cadre de réflexion commun dépassant les clivages entre différents milieux professionnels et sociaux et les divergences entre différents positionnements théoriques. Alors proclamer la nécessité du développement d'un tel cadre peut sembler aussi douteux que l'appel général de Holloway à " arrêter de fabriquer le capitalisme ». Il n'en reste pas moins que l'instauration de "l'activité vitale consciente » comme nouvelle base d'une vie en commun est forcément à ce prix, 
elle est impensable sans le développement, la diffusion et la réalisation consciente d'un tel changement.

INDEX

Mots-clés : critique du travail, correspondance, artistes, socialisme vert, travailleurs culturels, Wertkritik, Gorz André, dialogue, Vincent Jean-Marie, écologie, modèles alternatifs, Marx Karl, penser le Capital, ethos du travail, Eglise Orthodoxe, salariat, domination

\section{AUTEURS}

\section{DIETRICH HOSS}

Professeur des universités émérite, Université Lyon 2. 